\title{
Identification of Low-order Process Model with Time Delay from Closed-loop Step Test
}

\author{
Tao Liu, Furong Gao, Chunhui Zhao
}

\begin{abstract}
To facilitate control-oriented model identification during closed-loop system operation, a low-order model identification method is proposed in this paper, based on using closed-loop step response test. By introducing a damping factor to the closed-loop step response for realization of the Laplace transform, a frequency response estimation algorithm is developed in terms of the closed-loop control structure used for identification. Correspondingly, two model identification algorithms are derived analytically for obtaining the widely used low-order process models of first-order-plus-dead-time (FOPDT) and second-order-plus-dead-time (SOPDT), respectively. Illustrative examples from the recent literature are given to demonstrate the effectiveness of the proposed identification algorithms.
\end{abstract}

\section{INTRODUCTION}

A $\mathrm{S}$ model-based control methods have demonstrated apparently improved system performance for set-point tracking and load disturbance rejection in various industrial and chemical processes, control-oriented model identification methods have been increasingly explored in the process control community [1]-[4]. Among a variety of excitation signals for identification test, step response test has been widely practiced owing to its implementation simplicity. Most existing references have been devoted to identification methods based on open-loop step test(s). A few papers [5], [6] reported identification algorithms based on fitting several representative points in the process transient response to a step change. Bi et al. [7] gave a first-order-plus-dead-time (FOPDT) model fitting algorithm using numerical integral to the time domain expression of step response, which was further extended to obtain second-order-plus-dead-time (SOPDT) or higher order models [8], [9]. With a prescribed filter for LS fitting of the step response, Ahmed, Huang and Shah [10] presented an iterative procedure to determine the optimal time delay model. To guarantee identification accuracy against load disturbance or nonzero initial process conditions, modified or multiple step tests were proposed for development of robust identification methods [11]-[14]. For safety and economic reasons, many industrial processes are not allowed to be operated in an open-loop manner [1].

This work was supported in part by the Hong Kong Research Grants Council under Project No. 613107 and China National 973 Program (2009CB320603)

All the authors are with the Department of Chemical and Biomolecular Engineering, Hong Kong University of Science \& Technology, Clear Water Bay, Kowloon, Hong Kong (phone: 852-2358-7139; fax: 852-2358-0054; e-mail addresses: liurouter@ieee.org (T. Liu); kefgao@ust.hk (F. Gao); huihuizh@gmail.com (C. Zhao)).
Moreover, it has become more appealing for closed-loop identification test, in order to facilitate online tuning of the closed-loop controller [15]-[17]. Based on closed-loop step test in terms of the internal model control (IMC) structure, Häggblom K. E. [18] demonstrated that closed-loop identification facilitates better representation of the process dynamic response characteristics for closed-loop operation. Using a proportional $(\mathrm{P})$ or proportional-integral-derivative (PID) type controller for closed-loop step test, Wang and Cluett [19] developed an identification algorithm to obtain an continuous-time Laguerre model; Cai, Fang and Wang [20] reported a SOPDT identification algorithm from the analysis of closed-loop frequency response; Using first-order Taylor approximation for the time delays in individual channels, $\mathrm{Li}$ et al [21] presented a LS fitting algorithm for multivariable processes. Recent papers [22]-[24] developed closed-loop step identification methods for open-loop unstable processes. To describe the process dynamic characteristics with a suitable model structure, Piroddi and Leva [25] presented a step response classification method for model fitting. Using relay feedback to yield sustained oscillation of the closed-loop output, Padhy and Majhi [26] suggested an identification method based on the resulting limit cycle data. Besides, using the pseudo-random binary sequence (PRBS) as excitation to the process input or the set-point, closed-loop identification methods have also been reported for delay-free linear time-invariant processes [27-31]

In this paper, identification algorithms based on using a closed-loop step response test are proposed for obtaining low-order models of FOPDT and SOPDT to facilitate online tuning of time delay processes. By introducing a damping factor to the closed-loop step response for realization of the Laplace transform, an algorithm is first given to estimate the closed-loop frequency response. Accordingly, the process frequency response can be analytically derived from the closed-loop frequency response with the knowledge of the controller. In the sequel, two identification algorithms are analytically developed for obtaining FOPDT and SOPDT models, respectively. Both algorithms can give good fitting accuracy if the model structure adopted matches the real process.

\section{FREQUENCY RESPONSE ESTIMATION}

It is commonly known that the Fourier transform of a step response does not exist due to $\Delta y(t) \neq 0$ for $t \rightarrow \infty$, where $\Delta y(t)=y(t)-y\left(t_{0}\right)$ and $y\left(t_{0}\right)$ denotes the initial steady 
output, as shown in Fig.1. However, by substituting $s=\alpha+j \omega$ into the Laplace transform for the step response,

$$
\Delta Y(s)=\int_{0}^{\infty} \Delta y(t) e^{-s t} d t
$$

we can formulate

$$
\Delta Y(\alpha+j \omega)=\int_{0}^{\infty}\left[\Delta y(t) e^{-\alpha t}\right] e^{-j \omega t} d t
$$

Note that, if $\alpha>0$, there exists $\Delta y(t) e^{-\alpha t}=0$ for $t>t_{N}$, where $t_{N}$ may be numerically determined using the condition of $\Delta y\left(t_{N}\right) e^{-\alpha t_{N}} \approx 0$, since $\Delta y(t)$ reaches a steady value after the transient step response.

Therefore, by regarding $\alpha$ as a damping factor to the closed-loop step response for Laplace transform, we may compute $\Delta Y(\alpha+j \omega)$ from the $N$ points of step response data as

$$
\Delta Y(\alpha+j \omega)=\int_{0}^{t_{N}}\left[y(t) e^{-\alpha t}\right] e^{-j \omega t} d t
$$

For a closed-loop step test with initial steady state, i.e., $y(t)=r(t)=c$ for $t \leq t_{0}$, where $r(t)$ denotes the set-point value and $c$ is a constant, we may express the step change of the set-point by using a time shift of $t_{0}$ (i.e., letting $t_{0}=0$ ) as $\Delta r(t)= \begin{cases}0, & t \leq 0 \\ h, & t>0\end{cases}$

where $h$ is the magnitude of the step change. Its Laplace transform for $s=\alpha+j \omega$ with $\alpha>0$ can be analytically derived as

$\Delta R(\alpha+j \omega)=\int_{0}^{\infty} h e^{-(\alpha+j \omega) t} d t=\frac{h}{\alpha+j \omega}$

Hence, the closed-loop frequency response can be derived using (3) and (5) as

$T(\alpha+j \omega)=\frac{\alpha+j \omega}{h} \Delta Y(\alpha+j \omega), \alpha>0$

Note that $T(\alpha+j \omega) \rightarrow 0$ as $\alpha \rightarrow \infty$. On the contrary, $\alpha \rightarrow 0$ will cause $t_{N}$ much larger for computation of (6). A proper choice of $\alpha$ is therefore required for implementation. Considering that all the closed-loop transient response data to the step change should be used to ensure good estimation of the closed-loop frequency response, the following constraint is suggested to choose $\alpha$,

$$
\Delta y\left(t_{\text {set }}\right) e^{-\alpha t_{\text {set }}}>\delta
$$

where $\Delta y\left(t_{\text {set }}\right)$ denotes the steady-state output deviation to the step change in terms of the settling time $\left(t_{\text {set }}\right)$, and $\delta$ is a threshold of the computational precision that may be practically taken less than $1 \times 10^{-6}$. It follows from (7) that

$\alpha<\frac{1}{t_{\text {set }}} \ln \frac{\Delta y\left(t_{\text {set }}\right)}{\delta}$

To ensure computation efficiency with respect to the complex variable, $s=\alpha+j \omega$, for frequency response estimation, the lower bound of $\alpha$ may be simply taken larger than $\delta$, if there exists no limit on the time length of the step test.

Once $\alpha$ is chosen in terms of the above guideline, the time length, $t_{N}$, may be determined from a numerical constraint for computation of (3), i.e.,

$$
\Delta y\left(t_{N}\right) e^{-\alpha t_{N}}<\delta
$$

which can be solved as

$t_{N}>\frac{1}{\alpha} \ln \frac{\Delta y\left(t_{N}\right)}{\delta}$

Note that there exists the following Laplace transform for the initial steady state of the closed-loop system,

$L\left[\int_{0}^{t} \Delta y(t) d t\right]=\frac{\Delta Y(s)}{s}$

To guarantee identification robustness against measurement noise, we may compute the frequency response by

$$
T(\alpha+j \omega)=\frac{\frac{\Delta Y(\alpha+j \omega)}{\alpha+j \omega}}{\frac{\Delta R(\alpha+j \omega)}{\alpha+j \omega}}=\frac{(\alpha+j \omega)^{2}}{h} \int_{0}^{t_{N}}\left[\int_{0}^{t} \Delta y(\tau) d \tau\right] e^{-\alpha t} e^{-j \omega t} d t
$$

It can be seen from (12) that, rather than use individual output data measured from the step test, a time integral for each measurement point is used to compute the outer-layer integral for obtaining the frequency response estimation. This facilitates reducing measurement errors according to the statistic averaging principle.

Denote the $n$-th order derivative for a complex function of $F(s)$ with respect to $s$ as

$F^{(n)}(s)=\frac{d^{(n)}}{d s^{n}} F(s), n \geq 1$.

It follows from (3) and (6) that

$T^{(1)}(s)=\frac{1}{h} \int_{0}^{\infty}(1-s t) \Delta y(t) e^{-s t} d t$

$T^{(2)}(s)=\frac{1}{h} \int_{0}^{\infty} t(s t-2) \Delta y(t) e^{-s t} d t$

Hence, by letting $s=\alpha$ and choosing $\alpha$ as well as that for computation of (3), the time integral in (14) and (15) can be numerically computed. The corresponding time lengths of $t_{N}$ can be respectively determined using the numerical constraints,

$\left(1-\alpha t_{N}\right) \Delta y\left(t_{N}\right) e^{-\alpha t_{N}}<\delta$

$t_{N}\left(\alpha t_{N}-2\right) \Delta y\left(t_{N}\right) e^{-\alpha t_{N}}<\delta$

Without loss of generality, for a PID controller that is most commonly used in the closed-loop structure for a step test,

$C(s)=k_{\mathrm{C}}\left(1+\frac{1}{\tau_{\mathrm{I}} s}+\frac{\tau_{\mathrm{D}} s}{0.1 \tau_{\mathrm{D}} s+1}\right)$

where $k_{\mathrm{C}}$ denotes the controller gain, $\tau_{\mathrm{I}}$ the integral constant and $\tau_{\mathrm{D}}$ the derivative constant, it can be derived that

$C^{(1)}(s)=k_{\mathrm{C}}\left[-\frac{1}{\tau_{\mathrm{I}} s^{2}}+\frac{\tau_{\mathrm{D}}}{\left(0.1 \tau_{\mathrm{D}} s+1\right)^{2}}\right]$

$C^{(2)}(s)=2 k_{\mathrm{C}}\left[\frac{1}{\tau_{\mathrm{I}} s^{3}}-\frac{0.1 \tau_{\mathrm{D}}^{2}}{\left(0.1 \tau_{\mathrm{D}} s+1\right)^{3}}\right]$

Since the closed-loop transfer function can be derived as

$T(s)=\frac{G(s) C(s)}{1+G(s) C(s)}$

it follows that

$G(s)=\frac{T(s)}{C(s)[1-T(s)]}$ 
Accordingly, the first and second derivatives of (22) can be derived accordingly as

$$
\begin{aligned}
G^{(1)} & =\frac{T^{(1)} C+C^{(1)} T(T-1)}{C^{2}(1-T)^{2}} \\
G^{(2)} & =\frac{C T^{(2)}+2 C^{(1)} T^{(1)} T+C^{(2)} T(T-1)}{C^{2}(1-T)^{2}} \\
& -\frac{2\left[C T^{(1)}+C^{(1)} T(T-1)\right]\left[C C^{(1)}(1-T)-C^{2} T^{(1)}(1-T)\right]}{C^{3}(1-T)^{3}}
\end{aligned}
$$

Therefore, by substituting $s=\alpha+j \omega_{k}(k=1,2, \ldots, M$.$) ,$ where $M$ is the number of representative frequency response points in a user specified frequency range, the process frequency response can be numerically estimated for model fitting.

\section{Model IDENTIFICATION Algorithms}

Based on the above frequency response estimation algorithm, two algorithms are proposed herein for identification of the widely used FOPDT and SOPDT models, which are respectively in the form of

$$
\begin{aligned}
& G_{1}(s)=\frac{k_{\mathrm{p}}}{\tau_{\mathrm{p}} s+1} e^{-\theta s} \\
& G_{2}(s)=\frac{k_{\mathrm{p}}}{b_{2} s^{2}+b_{1} s+1} e^{-\theta s}
\end{aligned}
$$

where $k_{\mathrm{p}}$ denotes the process gain, $\theta$ the process time delay and $\tau_{\mathrm{p}}$ (or $b_{1}$ and $b_{2}$ ) the process time constant(s).

For clarity, the corresponding algorithms, Algorithm-I and Algorithm-II, are detailed in the following two subsections, respectively.

\section{A. Algorithm-I for FOPDT Model}

By regarding $s \in \mathbb{R}_{+}$and taking the natural logarithm for both sides of (25), we obtain

$$
\ln \left[G_{1}(s)\right]=\ln \left(k_{\mathrm{p}}\right)-\ln \left(\tau_{\mathrm{p}} s+1\right)-\theta s
$$

Subsequently, taking the first and second derivatives for both sides of (27) with respect to $s$ yields

$$
\begin{aligned}
& \frac{1}{G_{1}(s)} \frac{d}{d s}\left[G_{1}(s)\right]=-\frac{\tau_{\mathrm{p}}}{\tau_{\mathrm{p}} s+1}-\theta \\
& Q_{2}(s)=\frac{\tau_{\mathrm{p}}^{2}}{\left(\tau_{\mathrm{p}} s+1\right)^{2}}
\end{aligned}
$$

where $Q_{2}(s)=d\left[Q_{1}(s)\right] / d s$ and $Q_{1}(s)$ is the left side of (28).

Substituting $s=\alpha$ into (29), it can be derived that

$$
\tau_{\mathrm{p}}=\frac{\sqrt{Q_{2}}}{1-\alpha \sqrt{Q_{2}}}
$$

Consequently, the other two model parameters can be derived from (28) and (25) as

$$
\begin{aligned}
& \theta=-Q_{1}(\alpha)-\frac{\tau_{\mathrm{p}}}{\tau_{\mathrm{p}} \alpha+1} \\
& k_{\mathrm{p}}=\left(\tau_{\mathrm{p}} \alpha+1\right) G_{1}(\alpha) e^{\alpha \theta}
\end{aligned}
$$

Hence, the above algorithm named Algorithm-I for obtaining a FOPDT model can be summarized as:

(i) Choose $s=\alpha$ and $t_{N}$ to compute $T(\alpha), T^{(1)}(\alpha)$ and $T^{(2)}(\alpha)$ in terms of (6) (or (12)), (14) and (15);

(ii) Compute $C(\alpha), C^{(1)}(\alpha)$ and $C^{(2)}(\alpha)$ in terms of (18), (19) and (20);

(iii) Compute $G_{1}(\alpha), G_{1}^{(1)}(\alpha)$ and $G_{1}^{(2)}(\alpha)$ in terms of (22), (23) and (24);

(iv) Compute $Q_{1}(\alpha)$ and $Q_{2}(\alpha)$ in terms of (28) and (29);

(v) Compute the process time constant, $\tau_{\mathrm{p}}$, from (30);

(vi) Compute the process time delay, $\theta$, from (31);

(vii) Compute the process gain, $k_{\mathrm{p}}$, from (32).

\section{B. Algorithm-II for SOPDT Model}

Taking the natural logarithm for both sides of (26) yields

$\ln \left[G_{2}(s)\right]=\ln \left(k_{\mathrm{p}}\right)-\ln \left(b_{2} s^{2}+b_{1} s+1\right)-\theta s$

Accordingly, the first and second order derivatives for both sides of (33) with respect to $s$ can be derived respectively as

$$
\begin{aligned}
& \frac{1}{G_{2}(s)} \frac{d}{d s}\left[G_{2}(s)\right]=-\frac{2 b_{2} s+b_{1}}{b_{2} s^{2}+b_{1} s+1}-\theta \\
& Q_{2}(s)=\frac{2 b_{2}^{2} s^{2}+2 b_{2} b_{1} s+b_{1}^{2}-2 b_{2}}{\left(b_{2} s^{2}+b_{1} s+1\right)^{2}}
\end{aligned}
$$

where $Q_{2}(s)=d\left[Q_{1}(s)\right] / d s$ and $Q_{1}(s)$ is the left side of (34).

Substituting $s=\alpha$ into (35) yields

$$
\begin{aligned}
Q_{2}(\alpha)= & 2 b_{1}^{2}+b_{2}^{2}\left[2 \alpha^{2}-\alpha^{4} Q_{2}(\alpha)\right]+b_{1} b_{2}\left[2 \alpha-2 \alpha^{3} Q_{2}(\alpha)\right] \\
& -\left(b_{1}^{2}+2 b_{2}\right)\left[1+\alpha^{2} Q_{2}(\alpha)\right]-2 \alpha Q_{2}(\alpha) b_{1}
\end{aligned}
$$

To solve $b_{1}$ and $b_{2}$ from (36), we reformulate (36) in the LS form of

$\psi(\alpha)=\phi(\alpha)^{T} \gamma$

where

$$
\left\{\begin{array}{l}
\psi(\alpha)=Q_{2}(\alpha), \\
\phi(\alpha)=\left[2,2 \alpha^{2}-\alpha^{4} Q_{2}(\alpha), 2 \alpha-2 \alpha^{3} Q_{2}(\alpha),-1-\alpha^{2} Q_{2}(\alpha),-2 \alpha Q_{2}(\alpha)\right]^{T}, \\
\gamma=\left[b_{1}^{2}, b_{2}^{2}, b_{1} b_{2}, b_{1}^{2}+2 b_{2}, b_{1}\right]^{T} .
\end{array}\right.
$$

By choosing 5 different values of $\alpha$ in terms of the guideline of (8) and denoting $\Psi=\left[\psi\left(\alpha_{1}\right), \psi\left(\alpha_{2}\right), \ldots, \psi\left(\alpha_{5}\right)\right]^{T}$ and $\Phi=\left[\phi\left(\alpha_{1}\right), \phi\left(\alpha_{2}\right), \ldots, \phi\left(\alpha_{5}\right)\right]^{T}$, an LS solution can be derived from the linear regression,

$\gamma=\left(\Phi^{T} \Phi\right)^{-1} \Phi^{T} \Psi$

It obvious that all the columns of $\Phi$ are linearly independent with each other, such that $\Phi$ is guaranteed non-singular for computation of (39). Accordingly, there exists a unique solution of $\gamma$ for parameter estimation.

Then, the model parameters can be retrieved from $\gamma$ as

$\left\{\begin{array}{l}b_{1}=\sqrt{\gamma(1)} \\ b_{2}=\sqrt{\gamma(2)}\end{array}\right.$

Note that there exist three redundant fitting conditions in the parameter estimation of $\gamma$, which can be surely satisfied if the model structure matches the process to be identified. To procure fitting accuracy for a real high-order process, we may use $\gamma(3)$ and $\gamma(5)$ together with $\gamma(1)$ and $\gamma(2)$ to derive an 
LS fitting solution for parameter estimation in terms of using the natural logarithm, i.e.,

$\left[\begin{array}{ll}2 & 0 \\ 0 & 2 \\ 1 & 1 \\ 1 & 0\end{array}\right]\left[\begin{array}{l}\ln b_{1} \\ \ln b_{2}\end{array}\right]=\left[\begin{array}{l}\ln \gamma(1) \\ \ln \gamma(2) \\ \ln \gamma(3) \\ \ln \gamma(5)\end{array}\right]$

Consequently, the other two model parameters can be derived from (34) and (26) as

$$
\begin{aligned}
& \theta=-Q_{1}(\alpha)-\frac{2 b_{2} \alpha+b_{1}}{b_{2} \alpha^{2}+b_{1} \alpha+1} \\
& k_{\mathrm{p}}=\left(b_{2} \alpha^{2}+b_{1} \alpha+1\right) G_{2}(\alpha) e^{\alpha \theta}
\end{aligned}
$$

Hence, the above algorithm named Algorithm-II for obtaining an SOPDT model can be summarized as:

(i) Choose $s=\alpha$ and $t_{N}$ to compute $T(\alpha), T^{(1)}(\alpha)$ and $T^{(2)}(\alpha)$ in terms of (6) (or (12)), (14) and (15);

(ii) Compute $C(\alpha), C^{(1)}(\alpha)$ and $C^{(2)}(\alpha)$ in terms of (18), (19) and (20);

(iii) Compute $G_{2}(\alpha), G_{2}^{(1)}(\alpha)$ and $G_{2}^{(2)}(\alpha)$ in terms of (22), (23) and (24);

(iv) Compute $Q_{1}(\alpha)$ and $Q_{2}(\alpha)$ in terms of (34) and (35);

(v) Compute the time constants, $b_{1}$ and $b_{2}$, from (40) (or (41));

(vi) Compute the process time delay, $\theta$, from (42);

(vii) Compute the process gain, $k_{\mathrm{p}}$, from (43).

\section{ILLUSTRATION}

Example 1. Consider the FOPDT process studied in the recent literature [26],

$G(s)=\frac{1}{s+1} e^{-0.5 s}$

Based on relay feedback test with two P-type closed-loop controllers, Padhy and Majhi [26] derived a FOPDT model, $G_{\mathrm{m}}=1.0 e^{-0.5 s} /(0.9996 s+1)$. For illustration, the unity feedback control structure with a P-type controller of $k_{\mathrm{c}}=3.5$ that is equivalent to that of Padhy and Majhi [26], is used for a closed-loop step test with a step change of $h=0.5$ as in [26]. According to the guidelines given in (8) and (10), $\alpha=0.1$ and $t_{N}=200$ (s) are chosen to use the proposed Algorithm-I, resulting in a FOPDT model, $G_{\mathrm{m}}=1.0 e^{-0.5002 s} /(0.9998 s+1)$, which indicates very good accuracy.

To demonstrate identification robustness against measurement noise, assume that a random noise of $N\left(0, \sigma_{N}^{2}=0.94 \%\right)$, causing the noise-to-signal ratio (NSR) to $20 \%$, is added to the process output measurement which is then used for feedback control. By performing 100 Monte-Carlo tests in terms of varying the 'seed' of the noise generator from 1 to 100 , the identification results are obtained as

$$
G_{\mathrm{m}}=\frac{1.0003( \pm 0.006)}{0.9904( \pm 0.25) s+1} e^{-0.5012( \pm 0.042) s}
$$

where the model parameters are respectively the mean of 100 Monte-Carlo tests, and the values in the adjacent parentheses are the sample standard deviation.

Example 2. Consider the high-order process studied in the recent literature [32],

$$
G(s)=\frac{-s+1}{(6 s+1)(2 s+1)^{2}} e^{-s}
$$

By using an analytical model reduction method, Skogestad [32] gave a SOPDT model, $G_{\mathrm{m}}=1.0 e^{-3 s} /[(6 s+1)(3 s+1)]$, and correspondingly, a PID controller $k_{\mathrm{c}}=1.0, \tau_{\mathrm{I}}=6.0$ and $\tau_{\mathrm{D}}=3.0$ ) was tuned for closed-loop control. By performing a closed-loop step test with a unity step change in terms of the above PID controller, as shown in Fig.1, the proposed Algorithm-I using $\alpha=0.01$ and $t_{N}=1500(\mathrm{~s})$ gives a FOPDT model, $\quad G_{\mathrm{m}-1}=0.9992 e^{-6.6268 s} /(5.2148 s+1)$, corresponding to $e r r=6.39 \times 10^{-2}$ in terms of the closed-loop transient response in the time interval $[0,30] \mathrm{s}$. the proposed Algorithm-II based on the choice of $\alpha=0.01,0.11,0.21,0.21,0.41$ and $t_{N}=1200$ (s) gives a SOPDT model, $G_{\mathrm{m}-2}=0.9898 e^{-3.16 s} /\left(17.5077 s^{2}+8.6132 s+1\right)$, corresponding to err $=9.02 \times 10^{-5}$. Note that the SOPDT model of Skogestad [32] corresponds to err $=1.98 \times 10^{-4}$. The Nyquist plots of these SOPDT models are shown in Fig.2. It is seen that the frequency response of the proposed SOPDT model almost overlaps with that of the real process, which may facilitate better control performance, according to the model-based PID tuning method of Skogestad [32].

\section{CONCLUSION}

Control-oriented low-order model identification methods have been increasingly appealed for improving control system design and online autotuning in engineering practices. By introducing a damping factor to the closed-loop step response for realization of the Laplace transform, a frequency response estimation algorithm has been proposed for model fitting. Accordingly, two model identification algorithms have been analytically developed for practical applications. Both of the proposed algorithms can give good accuracy if the model structure adopted matches the process to be identified. Two illustrative examples from the recent literature have demonstrated the effectiveness of the proposed algorithms.

\section{REFERENCES}

[1] D. E. Seborg, T. F. Edgar, and D. A. Mellichamp, Process Dynamics and Control, 2nd ed. Hoboken, NJ: John Wiley \& Sons., 2003.

[2] K. J. Åström and T. Hägglund, Advanced PID Control. Research Triangle Park, NC: ISA Society of America, 2005.

[3] H. Garnier and L. Wang (ed), Identification of Continuous-time Models from Sampled Data. London: Springer-Verlag, 2008.

[4] T. Liu and F. Gao, "A systematic approach for on-line identification of second-order process model from relay feedback test," AIChE J., vol.54, pp.1560-1578, 2008.

[5] G. P. Rangaiah and P. R. Krishnaswamy, "Estimating second-order dead time parameters from underdamped process transients," Chemical Engineering Science, vol.51, pp.1149-1155, 1996. 
[6] H. P. Huang, M. W. Lee, and C. L. Chen, "A system of procedures for identification of simple models using transient step response," Ind. Eng. Chem. Res., vol.40, pp.1903-1915, 2001.

[7] Q. Bi, W. J. Cai, E. L. Lee, Q. G. Wang, C. C. Hang, and Y. Zhang, "Robust identification of first-order plus dead-time model from step response," Control Engineering Practice, vol.7, pp.71-77, 1999.

[8] Q. G. Wang and Y. Zhang, "Robust identification of continuous systems with dead-time from step responses," Automatica, vol.37, pp.377-390, 2001.

[9] Q. G. Wang, X. Guo, and Y. Zhang, "Direct identification of continuous time delay systems from step responses," J. Process Control, vol.11, pp.531-542, 2001.

[10] S. Ahmed, B. Huang, and S. L. Shah, "Novel identification method from step response," Control Engineering Practice, vol.15, pp.545-556, 2007.

[11] T. Liu and F. Gao, "Robust step-like identification of low-order process model under nonzero initial conditions and disturbance," IEEE Trans. Automat. Contr., vol.53, pp.2690-2695, 2008.

[12] S. Ahmed, B. Huang, and S. L. Shah, "Identification from step responses with transient initial conditions," J. Process Control, vol.18, pp.121-130, 2008.

[13] M. Liu, Q. G. Wang, B. Huang, and C. C. Hang, "Improved identification of continuous-time delay processes from piecewise step tests," J. Process Control, vol.17, pp.51-57, 2007.

[14] S. H. Hwang and S. T. Lai, "Use of two-stage least-squares algorithms for identification of continuous systems with time delay based on pulse responses," Automatica, vol.40, pp.1561-1568, 2004.

[15] I. D. Landau, "Identification in closed loop: a powerful design tool (better design models, simpler controllers)," Control Engineering Practice, vol.9, pp.51-65, 2001.

[16] M. Ohshima, "Control and design problems in material processing - How can process systems engineers contribute to material processing?," J. Process Control, vol.13, pp.599-605, 2003.

[17] N. F. Thornhill, S. C. Patwardhan, and S. L. Shah, "A continuous stirred tank heater simulation model with applications," J. Process Control, vol.18, pp.347-360, 2008.

[18] K. E. Häggblom, "Combined internal model and inferential control of a distillation column via closed-loop identification," J. Process Control, vol.6, pp.223-232, 1996;

[19] L. Wang and W. R. Cluett, "System identification based on closed-loop step response data," IET Control Theory \& Appl., vol.141, pp.107-110, 1994.

[20] W. J. Cai, Y. Fang, and Y. G. Wang, "Robust closed-loop process identification from step test," in the 8th International Conference on Control, Automation, Robotics and Vision, Kunming, China, 2004, pp.907-912.

[21] S. Y. Li., W. J. Cai, H. Mei, and Q. Xiong, "Robust decentralized parameter identification for two-input two-output process from closed-loop step responses," Control Engineering Practice, vol.13, pp. 519-531, 2005.

[22] P. N. Paraskevopoulos, G. D. Pasgianos, and K. G. Arvanitis, "New tuning and identification methods for unstable first order plus dead-time," IEEE Trans. Control Systems Technology, vol.12, pp.455-464, 2004.

[23] R. P. Sree and M. Chidambaram, "Improved closed loop identification of transfer function model for unstable systems," Journal of the Franklin Institute, vol.343, pp.152-160, 2006.

[24] E. Cheres, "Parameter estimation of an unstable system with a PID controller in a closed loop configuration," Journal of the Franklin Institute, vol.343, pp.204-209, 2006.

[25] L. Piroddi and A. Leva, "Step response classification for model-based autotuning via polygonal curve approximation," J. Process Control, vol.17, pp.641-652, 2007.

[26] P. K. Padhy and S. Majhi, "Relay based PI-PD design for stable and unstable FOPDT processes," Computers and Chemical Engineering, vol.30, pp.790-796, 2006.

[27] B. Huang and S. L. Shah, "Closed-loop identification: a two step approach," Journal of Process Control, vol.7, pp.425-438, 1997.

[28] A. Esmaili, J. F. MacGregor, and P. A. Taylor, "Direct and two-step methods for closed-loop identification: a comparison of asymptotic and finite data set performance," J. Process Control, vol.10, pp.525-537, 2000 .
[29] H. Garnier, M. Gilson, and W. X. Zheng, "A bias-eliminated least-squares method for continuous-time model identification of closed-loop systems," Int. J. Control, vol.73, pp.38-48, 2000.

[30] S. W. Sung, S. Y. Lee, H. J. Kwak, and I.-B. Lee, "Continuous-time subspace system identification method," Ind. Eng. Chem. Res., vol.40, pp.2886-2896, 2001.

[31] D. R. Saffer and F. J. Doyle, "Closed-loop identification with MPC for an industrial scale CD-control problem," IET Control Theory \& Appl., vol.149, pp.448-456, 2002.

[32] S. Skogestad, "Simple analytical rules for model reduction and PID controller tuning," J. Process Control, vol.13, pp.291-309, 2003.

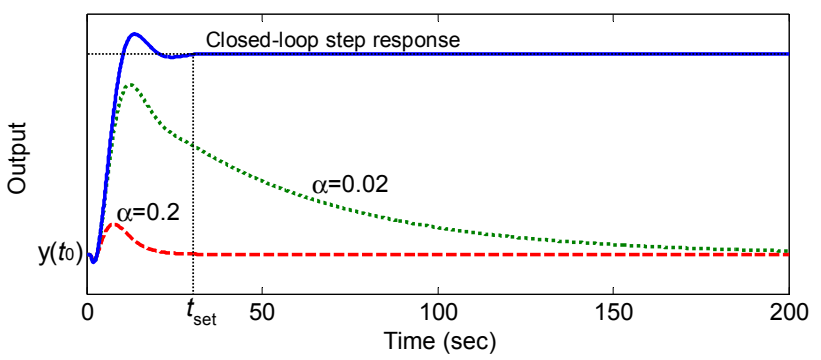

Fig. 1. Illustration of choosing $\alpha$ for closed-loop step test

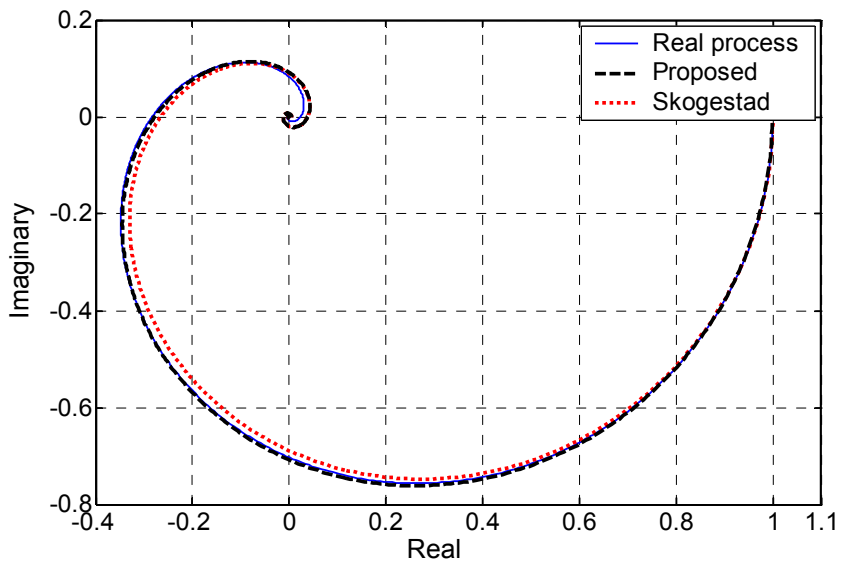

Fig. 2. Nyquist plots of identified SOPDT models for example 2 